\title{
Interactions of an Arabidopsis RanBPM homologue with LisH-CTLH domain proteins revealed high conservation of $\mathrm{CTLH}$ complexes in eukaryotes
}

Eva Tomaštíková', Věra Cenklová², Lucie Kohoutová3 ${ }^{3}$ Beáta Petrovská1, Lenka Váchová2, Petr Halada³, Gabriela Kočárová ${ }^{3}$ and Pavla Binarová ${ }^{3^{*}}$

\begin{abstract}
Background: RanBPM (Ran-binding protein in the microtubule-organizing centre) was originally reported as a centrosome-associated protein in human cells. However, RanBPM protein containing highly conserved SPRY, LisH, CTLH and CRA domains is currently considered as a scaffolding protein with multiple cellular functions. A plant homologue of RanBPM has not yet been characterized.

Results: Based on sequence similarity, we identified a homologue of the human RanBPM in Arabidopsis thaliana. AtRanBPM protein has highly conserved SPRY, LisH, CTLH and CRA domains. Cell fractionation showed that endogenous AtRanBPM or expressed GFP-AtRanBPM are mainly cytoplasmic proteins with only a minor portion detectable in microsomal fractions. AtRanBPM was identified predominantly in the form of soluble cytoplasmic complexes 230 - $500 \mathrm{kDa}$ in size. Immunopurification of AtRanBPM followed by mass spectrometric analysis identified proteins containing LisH and CRA domains; LisH, CRA, RING-U-box domains and a transducin/WD40 repeats in a complex with AtRanBPM. Homologues of identified proteins are known to be components of the C-terminal to the LisH motif (CTLH) complexes in humans and budding yeast. Microscopic analysis of GFP-AtRanBPM in vivo and immunofluorescence localization of endogenous AtRanBPM protein in cultured cells and seedlings of Arabidopsis showed mainly cytoplasmic and nuclear localization. Absence of colocalization with $\gamma$-tubulin was consistent with the biochemical data and suggests another than a centrosomal role of the AtRanBPM protein.
\end{abstract}

Conclusion: We showed that as yet uncharacterized Arabidopsis RanBPM protein physically interacts with LisH-CTLH domain-containing proteins. The newly identified high molecular weight cytoplasmic protein complexes of AtRanBPM showed homology with CTLH types of complexes described in mammals and budding yeast. Although the exact functions of the CTLH complexes in scaffolding of protein degradation, in protein interactions and in signalling from the periphery to the cell centre are not yet fully understood, structural conservation of the complexes across eukaryotes suggests their important biological role.

Keywords: Arabidopsis homologue of RanBPM, CTLH-complex, LisH-CTLH domain proteins

\footnotetext{
* Correspondence: binarova@biomed.cas.cz

${ }^{3}$ Institute of Microbiology, AS CR, v.v.i., Vídeňská 1083, 142 20, Prague 4,

Czech Republic

Full list of author information is available at the end of the article
} 


\section{Background}

Ras-related nuclear proteins (Rans) are abundant small GTPases, associated with Ran-specific nuclear GEFs (guanine nucleotide exchange factors), cytoplasmic GAPs (GTPase activating proteins), and with RanBP1 (Ran binding protein 1) that stimulate RanGTP hydrolysis in the cytoplasm [1,2]. The Arabidopsis genome contains three genes encoding AtRan [3], two genes encoding AtRanGAP related proteins [4] and three genes for RanBP1 isoforms - RanBP1a, RanBP1b and RanBP1c $[3,5]$. Plant Ran binding proteins (RanBPs) display significant homology with yeast and mammalian RanBPs, but there is little evidence for their biological function $[6,7]$.

One RanBP in animal cells, RanBPM (RanBP9), was identified in a yeast two-hybrid screen with Ran as a bait. RanBPM comprises four domains - SPRY, LisH, CTLH and CRA and is homologous to the human RanBP10 protein [8]. Although RanBPM and RanBP10 have been shown to bind the Ran protein, they do not contain a consensus Ran-binding sequence [9]. RanBPM was defined as a member of the Scorpin family of proteins (SPRY-containing Ran binding protein) with a unique domain organization [10]. As reviewed in Suresh et al. [11], numerous protein interactions described for the RanBPM protein suggest its multiple roles in the regulation of protein stability, cell cycle regulation and other as yet undefined cellular processes.

RanBPM was reported to be a part of the large CTLH (C-terminal to the LisH motif) complexes [12-14]. CTLH complexes composed of LisH, CTLH and CRA domain containing proteins, transducin/WD40 repeat proteins, and armadillo repeat proteins have been found in mammals and yeast $[15,16]$. Mammalian and yeast CTLH complexes are structurally conserved but their biological function is still not fully understood. In yeast, the CTLH complex of Gid/Vid proteins plays a role in vacuole and proteasome-dependent fructose-1,6-bisphosphatase degradation [16]. Similarly, it has been suggested that CTLH complexes partake in lysosome and proteasome-dependent proteolysis in mammalian cells [17]. Data on proteins with SPRY, LisH, CTLH or CRA domain-containing proteins in plants are limited. In Pinus radiata, the SPRY domain containing protein, SEPR11, is the homologue of a Trithoraxgroup member and is involved in plant reproduction and development [18]. In Oryza sativa, the LisH domaincontaining protein, OsLIS-L1 is required for male gametophyte formation and the first internode elongation [19].

Here we provide data on an Arabidopsis homologue of RanBPM that belongs to the uncharacterized family of plant SPRY, LisH, CTLH and CRA domain-containing proteins. We used in silico analysis, biochemical, proteomic and microscopic analyses in vivo and in situ to characterize AtRanBPM. We found that the AtRanBPM protein is present predominantly in the form of large cytoplasmic protein complexes that are structurally homologous to the CTLH type of complexes described in mammals and budding yeast.

\section{Results}

The Arabidopsis homologue of RanBPM is a SPRY-domain containing protein

By homology search of the Arabidopsis thaliana genome, we found a SPRY-domain containing protein AtRanBPM (At1g35470), which is a homologue of the human RanBPM (RanBP9) protein. The AtRanBPM cDNA contains a single open reading frame and consists of 467 amino acids. AtRanBPM is a member of the HOM002780 gene family that comprises 44 genes in 21 plant species, particularly from the ORTHO002658 subfamily. In Arabidopsis there are three paralogues of AtRanBPM (At4g09310, At4g09200, At4g09250) and one gene originating from the segmental duplication of chromosome 1 (At4g09340) (Plaza 2.0; A resource for plant comparative genomics, [20]). The products of these genes are described as SPRY domain-containing proteins but their biological roles in plants have not yet been identified.

The AtRanBPM protein is composed of four domains - SPRY, LisH, CTLH and CRA (Figure 1A). SPRY, LisH, CTLH and CRA domain-containing proteins are widely spread across eukaryotes. The protein encoded by AtRanBPM is annotated as a protein of unknown function (TAIR; The Arabidopsis Information Resource, [21]). As shown in Figure 1B, AtRanBPM has close homologues in other plant species such as Ricinus communis (67\% identities, $79 \%$ positives), Vitis vinifera (66\%, $78 \%)$, Populus trichocarpa (67\%, 80\%), Sorghum bicolor (57\%, 74\%), Zea mays (57\%, 74\%) and Oryza sativa Japonica group (57\%, 73\%) (Figure 1B, Additional file 1). The Arabidopsis RanBPM shows a close identity and similarity to its SPRY, LisH, CTLH and CRA domains with mammalian RanBPM and RanBP10 (Figure 1C, Additional file 2) (WU-BLAST, Basic Local Alignment Search Tool, [22]). However, there is an insertion of about 150 amino acids between the CTLH and CRA domains in human proteins (Figure 1C). A phylogenetic analysis performed using MEGA5 software (MEGA 5.02, Molecular Evolutionary Genetics Analysis, [23]) revealed that Arabidopsis RanBPM grouped with homologues from other plant species. The metazoan RanBPM homologues grouped in a separated clade. The Saccharomyces homologue, Gid1/Vid30, appears to be ancestral to the plant and metazoan clades (Additional file 3).

SPRY, LisH, CTLH and CRA conserved domains are mainly involved in protein-protein interactions. The SPRY domain (SPla and the RYanodine Receptor) was originally identified as a structural motif in ryanodine receptors [24]. Proteins with SPRY domains are involved in intracellular calcium release, as is the case for ryanodine 


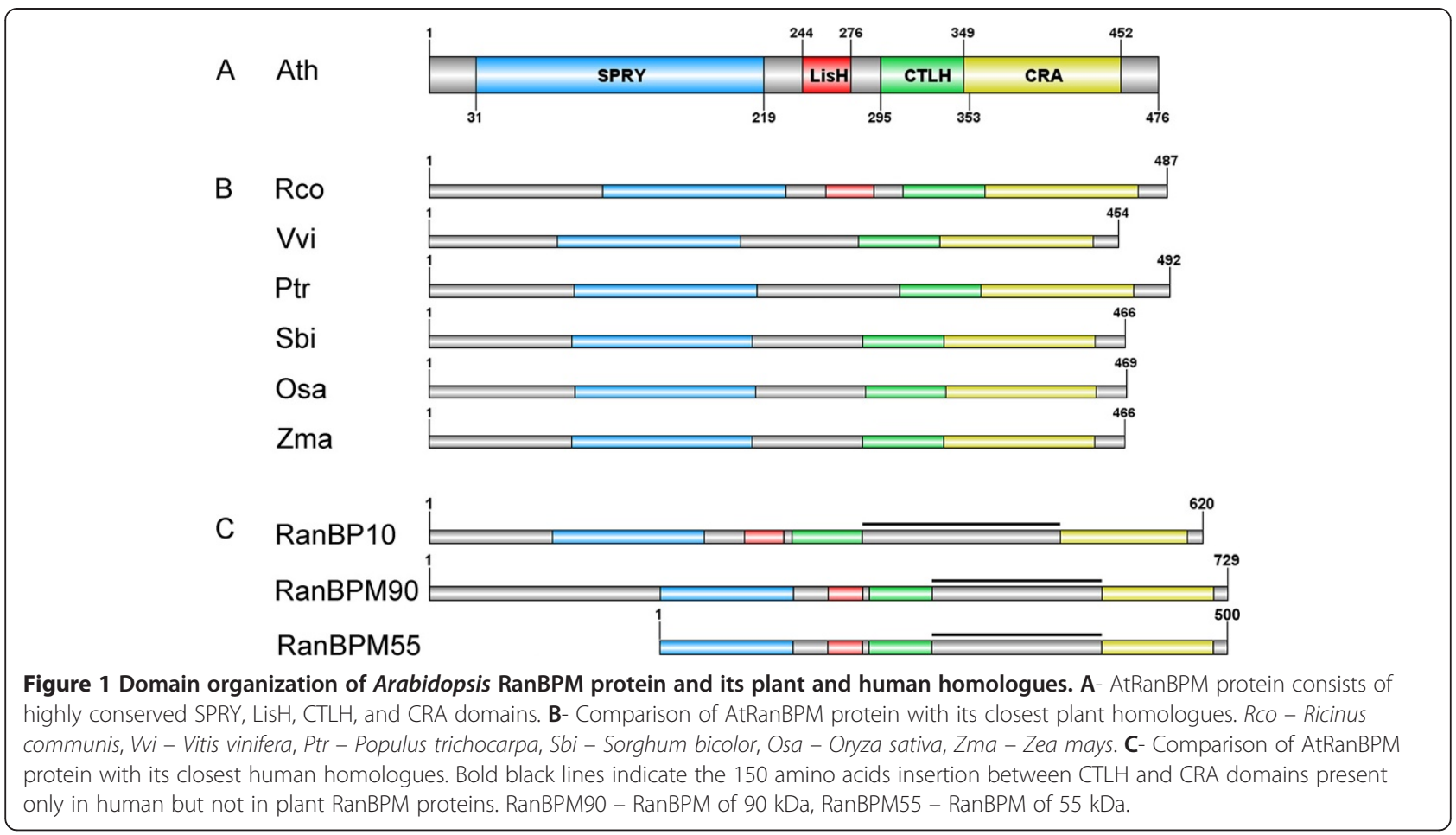

receptors, in RNA metabolism and in regulatory and developmental processes [25]. The LisH domain (Lissencephaly type-1-like homology motif) functions as a stable homodimerization and dimerization motif and its contribution to the dynamics of microtubules has been suggested [26]. Located adjacent to the C-terminus of the LisH domain is a C-terminal to the LisH motif (CTLH) that has a putative $\alpha$-helical structure with an as yet undescribed function. The CRA domain (CT11-RanBPM) was found to be a motif in the C-terminal part of RanBPM and RanBP10 and represents a protein-protein interaction domain often found in Ran-binding proteins [27].

Putative interaction sites of AtRanBPM were analysed using ELM (The Eukaryotic Linear Motif for functional sites in proteins, [28]) and SUMOsp 2.0 databases (SUMOylation Sites Prediction, [29]) (Figure 2). There are common motifs in the AtRanBPM amino acid sequence, such as phosphorylation consensus sites for phosphoinositide-3-OH kinase related kinases, a protein kinase A phosphorylation site, a mitogen-activated protein kinase phosphorylation site and docking motif, and a putative cyclin recognition site. Further, the AtRanBPM amino acid sequence possesses forkhead-associated phosphopeptide binding domains and a Y-based sorting signal which might act in endosomal and secretory pathways.

\section{Protein expression levels, cellular distribution and molecular forms of AtRanBPM}

The AtRanBPM coding sequence [EMBL:AEE31799] was cloned into Gateway-compatible plant binary vectors for
N-terminal GFP and C-terminal GFP fusion. Arabidopsis cell suspension cultures were transformed according to Mathur et al. [30]. Transformed Arabidopsis plants were obtained through the floral-dip method [31].

An antibody against a peptide from the C-terminal part of the AtRanBPM molecule was produced in rabbits and affinity purified using the immunogenic peptide. When cell lines expressing GFP-AtRanBPM were analysed by Western blotting of separated Arabidopsis extracts, the antibody recognized a band of $52 \mathrm{kDa}$, the predicted molecular mass (MW) of AtRanBPM, and a band corresponding to the MW of GFP-AtRanBPM (Figure 3A). Western blot further showed that the protein levels of AtRanBPM were much lower in Arabidopsis seedlings than cell cultures. In cultured Arabidopsis cells, there was no significant difference in AtRanBPM protein levels between dividing cells and stationary growing non-dividing cells (Figure 3B). Similarly, there was no difference in AtRanBPM protein levels between four, eight and 15 day old Arabidopsis seedlings. Our data correspond to those on AtRanBPM expression found in the publically available Affymetrix expression databases, the Arabidopsis eFP browser [32], and the Genevestigator database [33] where AtRanBPM has a constant level of expression in analysed tissues as well as during seedling development.

The cellular distribution of AtRanBPM was analysed using differential centrifugation. As shown in Figure 3C, the majority of the protein was cytoplasmic and only small amounts sedimented either in low speed pellets 


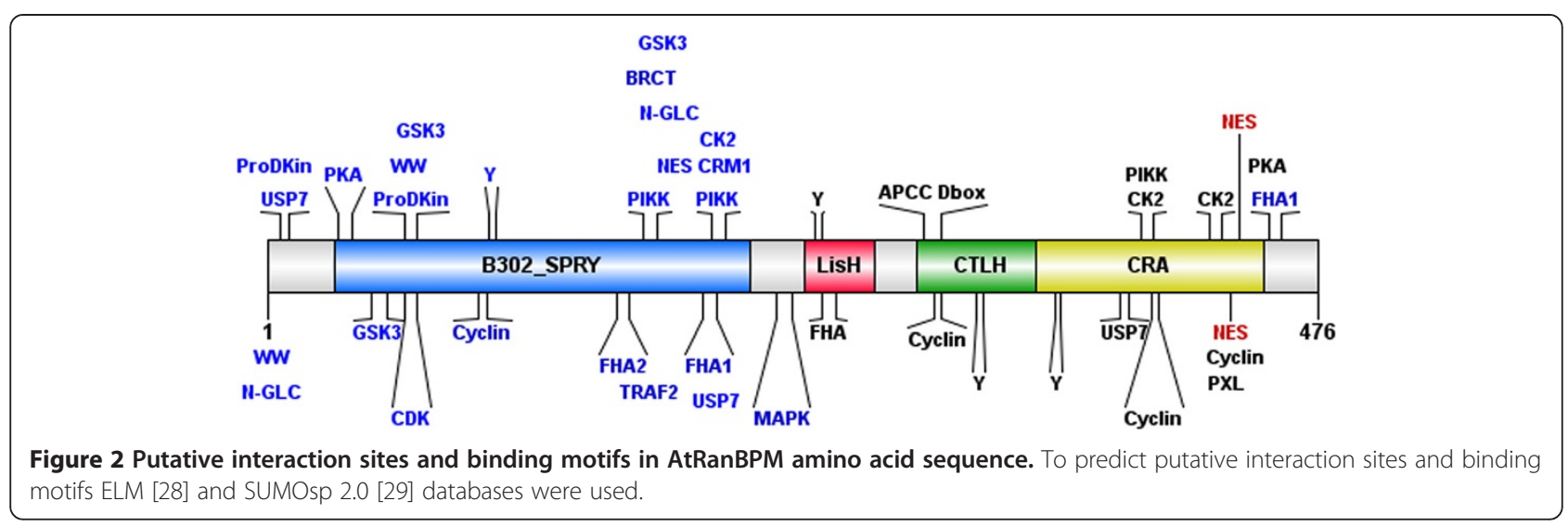

or in the microsomal pellet (P100). The fact that AtRanBPM was also sedimented from a high-speed cytoplasmic S100 extract to pellet P200 indicated that the protein might be present in the form of higher MW complexes. The separation of S100 high speed extract by native polyacrylamide gel electrophoresis (native PAGE) confirmed cell fractionation data and suggested that AtRanBPM is a component of protein complexes with a molecular mass of around $\sim 230-500 \mathrm{kDa}$ (Figure 3D). Further, we immunopurified GFP-AtRanBPM using the GFP trap. Separation of purified GFP-AtRanBPM by native PAGE followed by Western blotting showed the
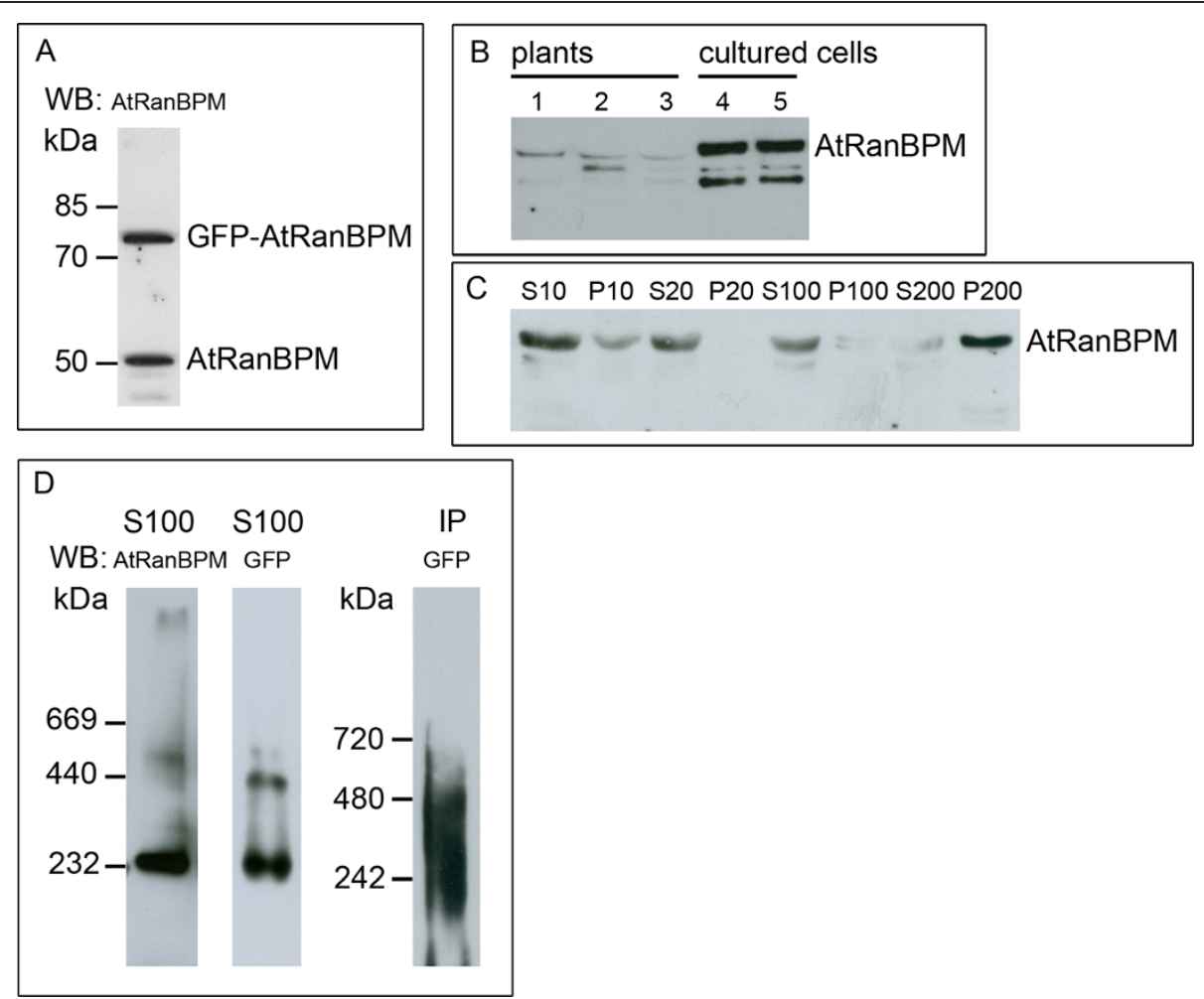

Figure 3 Cellular distribution of AtRanBPM and AtRanBPM complexes. A- The antibody against AtRanBPM recognizes a band of $52 \mathrm{kDa}$, which corresponds to endogenous AtRanBPM protein, and a band of $80 \mathrm{kDa}$, which corresponds to expressed GFP-AtRanBPM from cultured cells expressing GFP-AtRanBPM. B- Comparison of protein levels of AtRanBPM in Arabidopsis seedlings and cultured cells. Lines 1, 2, and 3-fractions S10 from four-, eight-, and 15-d-old seedlings; lines 4 and 5 - fractions S10 from exponentially growing cultured cells (3 days after subculture) and stationary phase cultured cells ( 6 days after subculture). Per line, $25 \mu \mathrm{g}$ of protein content of S10 fraction was loaded. C- Distribution of AtRanBPM in the cellular fractions of Arabidopsis cultured cells. The majority of the protein was present in cytoplasm (fractions S10, S20, S100), a small amount of the protein was found in pellet P10. AtRanBPM was spun down to high-speed pellet P200 probably in the form of complexes. D- GFP-AtRanBPM complexes from high-speed supernatant S100 and from immunoprecipitate (IP) were resolved by non-denaturing PAGE and detected by anti-AtRanBPM antibody or anti-GFP antibody as indicated. For S100 fractions, 15 and $10 \mu \mathrm{g}$ of protein content was loaded. 
presence of complexes of similar size as observed when high-speed supernatant S100 was analysed (Figure 3D). Altogether these data showed that AtRanBPM is present predominantly in the form of large cytoplasmic complexes.

\section{Analysis of AtRanBPM protein complexes} by mass spectrometry

To analyse putative interactors of AtRanBPM in the complexes, GFP-AtRanBPM and copurified proteins were separated by SDS-PAGE followed by silver staining, and specific bands were excised and analysed by MALDI-MS (Figure 4A). GFP immunopurification performed in extracts from wild type cell was used as a negative control. Neither AtRanBPM protein nor bands corresponding to the MW of proteins copurified with GFP-AtRanBPM were observed in the negative controls and only background contamination such as HSPs and cytoskeletal proteins were detected by MALDI-MS (Additional file 4). We found that proteins reproducibly copurifying with AtRanBPM belong to CTLH-domain containing proteins including LisH (At1g61150), CRA and U-box (At3g55070, At4g37880), and to the transducin/WD-40 domain-containing proteins At5g08560, and At5g43920 (Table 1, Figure 4B). Alternatively to MALDI-MS, we used LC-MALDI-MS/ MS analysis of eluates from the GFP trap to confirm the interaction of GFP-AtRanBPM with the proteins mentioned above.

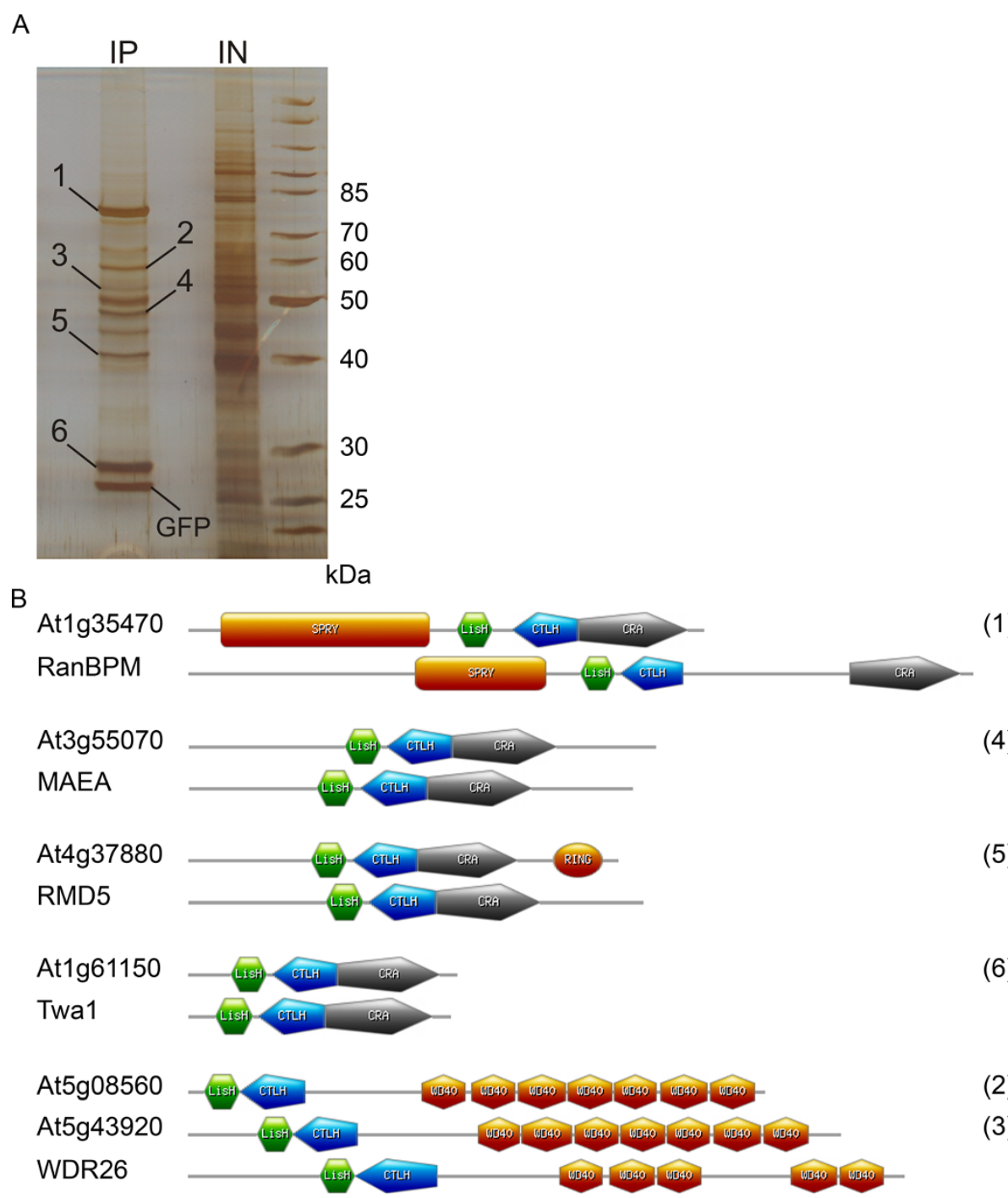

Figure 4 Proteins of CTLH complexes copurified with AtRanBPM from Arabidopsis extracts. A- Affinity purification of GFP-AtRanBPM from the input of S20 of GFP-AtRanBPM culture (IN) using GFP-Trap A beads. Proteins were eluted from affinity beads (IP: anti-GFP) and run on SDS gel electrophoresis with silver staining detection. MALDI-MS analysis identified among proteins copurified with AtRanBPM the plant homologues of proteins that form CTLH complex in human cells. B- Comparison of proteins copurified with AtRanBPM (numbers in brackets present number of band shown in Figure 4A) with their counterparts - the proteins of human CTLH complex. 
Table 1 Proteins copurified with AtRanBPM are members of CTLH complexes

\begin{tabular}{|c|c|c|c|c|c|c|c|}
\hline Protein name & AGI number & $\begin{array}{c}\text { Band } \\
\text { No. }\end{array}$ & $\begin{array}{c}\mathrm{MW} \\
{[\mathrm{kDa}]}\end{array}$ & $\begin{array}{c}\text { No. } \\
\text { peptides }\end{array}$ & $\begin{array}{c}\text { Sequence } \\
\text { coverage [\%] }\end{array}$ & $\begin{array}{l}\text { Aranet/ } \\
\text { ATTED-II }\end{array}$ & $\begin{array}{l}\text { Members of CTLH complex } \\
\text { in other organisms }\end{array}$ \\
\hline AtRanBPM & At1g35470 & 1 & 52 & $17 / 41$ & $41 / 60$ & & RanBPM [Q96S59] Gid1/Nid30 [P53076]*** \\
\hline LisH and RanBPM domain-containing protein & At1g61150 & 6 & 25 & $8 / 26$ & $41 / 82$ & $-/+$ & Twa1 [Q9NWU2]**Gid8 [P40208] $]^{* * *}$ \\
\hline LisH/CRA/RING-U-box domain-containing protein & At3g55070 & 4 & 48 & $10 / 17$ & $28 / 31$ & $+/-$ & MAEA [Q7L5Y9]*Gid9p [P40492] ${ }^{* * *}$ \\
\hline LisH/CRA/RING-U-box domain-containing protein & At4g37880 & 5 & 44 & $9 / 8$ & $32 / 22$ & $+/-$ & RMD5 [Q9H871]*Gid2 [Q12508]*** \\
\hline Transducin/WD40 domain-containing protein & At5g08560 & 2 & 66 & $17 / 14$ & $38 / 31$ & $+/-$ & WDR26 [Q9H7D7] \\
\hline Transducin/WD40 domain-containing protein & At5g43920 & 3 & 60 & $6 / 4$ & $13 / 7$ & $+/-$ & WDR26 [Q9H7D7] \\
\hline
\end{tabular}

Proteins (for band numbering see Figure 4A) copurified with AtRanBPM protein were identified by both MALDI-MS and LC-MALDI-MS/MS analysis. The values before and after the slash in the columns "No. peptides" and "Sequence coverage" refer to MALDI-MS and LC-MALDI-MS/MS data, respectively. Homologues of human CTLH-complex members reported by Kobayashi et al., [15] (*), proteins in the complex described by Umeda et al. [12] (**), yeast homologues in the Gid complex published by Regelmann et al. [16] (**). 

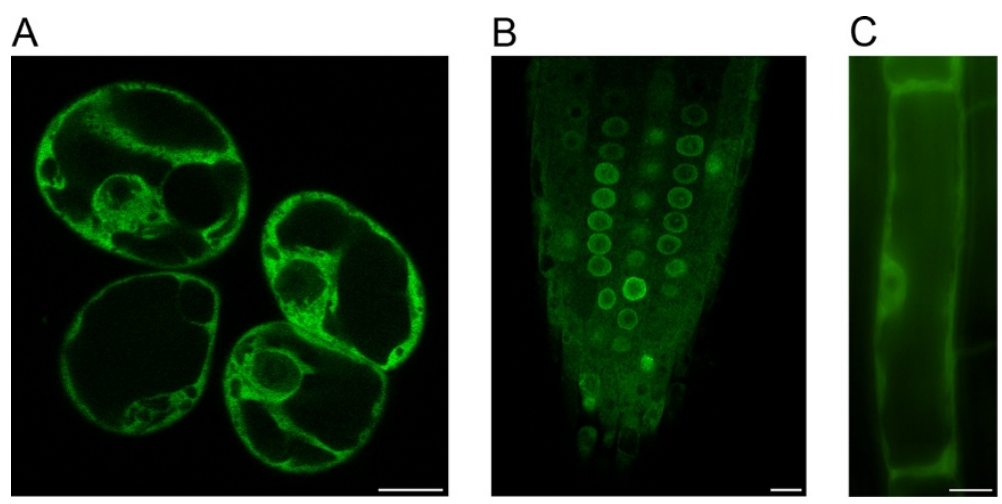

Figure $\mathbf{5}$ In vivo localization of GFP-AtRanBPM in Arabidopsis cells and seedlings. A- Localization of GFP-AtRanBPM in Arabidopsis suspension culture. The protein is localized in the cytoplasm and in the perinuclear area. Weaker signal is present in nuclei. B- Root tip of 8 days old Arabidopsis plant. Weak cytoplasmic signal for GFP-AtRanBPM is enriched in the perinuclear area. C-Cytoplasmic and perinuclear localization of GFP-AtRanBPM in differentiated cells of root. Bars $=10 \mu \mathrm{m}$.

Database search indicated that the proteins copurified with AtRanBPM were homologous to protein components of the human CTLH complex [15]. The CTLH complex is annotated in CORUM (The comprehensive resource for mammalian complexes, [34]) as a complex with a putative function in regulating cell migration, nucleokinesis, chromosome segregation and microtubule dynamics. Components of the CTLH complex are conserved amongst animal species and their presence is predicted for other eukaryotes such as plants and fungi. A relatively lower degree of homology with individual mammalian and plant members of CTLH complexes (Figure 4B, Additional file 5) is due to the fact that part of the molecule separating highly conserved domains contains insertions and deletions of various lengths, differing among plant, mammalian and/or yeast homologues.

The LC-MALDI-MS/MS analysis also identified among proteins copurified with AtRanBPM Armadillo-repeatcontaining protein (At3g08947) (Additional file 6). However, the protein was not proven to be a homologue of the human armadillo repeat ARMC8, a member of the CTLH complex $[34,35]$. Further, Yippee family proteins (At5g53940, At2g40110) and Yippee-like protein (At3g08890) were copurified with AtRanBPM and identified by LC-MALDI-MS/MS (Additional file 6). Yippeelike proteins belong to the YPEL family of proteins whose members have been described as interactors of the human RanBPM [10]. Proteins At5g08560, At5g43920, At3g55070, and At3g37880, copurified with AtRanBPM were predicted to interact within a functional network in the Aranet database (Probabilistic Functional Gene Network for Arabidopsis thaliana, [36]) (Table 1).

Together these data suggest that plant AtRanBPM protein, like its yeast and mammalian homologues, is a component of a cytoplasmic multiprotein complex where it interacts with LisH-CTLH domain-containing proteins and armadillo-repeat-containing proteins.
In vivo localization of GFP-AtRanBPM and AtRanBPM immunofluorescence labelling in Arabidopsis cultured cells and seedlings

In cultured cells of Arabidopsis, the GFP-AtRanBPM signal was localized in the cytoplasm and in the perinuclear area of interphase cells, with a weaker signal detected in nuclei (Figure 5A). We observed no gainof-function phenotype of Arabidopsis seedlings expressing GFP-AtRanBPM from Gateway vectors. Similarly to cultured cells, the GFP-AtRanBPM signal was localized in the cytoplasm, in nuclei and accumulated in the vicinity of nuclei in dividing zone of roots (Figure 5B). In differentiated root cells, GFP-AtRanBPM was cytoplasmic and in the perinuclear area (Figure 5C, Additional file 7). C-terminal GFP fusions showed a similar localization pattern when expressed transiently in cultured cells of Arabidopsis (Additional file 8).

Immunofluorescence analyses showed that the AtRanBPM protein was distributed patchily in the cytoplasm and nuclei; in non-dividing cells it accumulated in the perinuclear region and in dividing cells, the signal was slightly enriched in the area of the mitotic spindle and the phragmoplast (Figure 6A). Further we compared localization of the endogenous protein and GFPAtRanBPM by double labelling with anti-AtRanBPM and anti-GFP antibodies. We confirmed a similar localization pattern for AtRanBPM and expressed AtRanBPM with a cytoplasmic signal slightly enriched in the perinuclear area and a weaker nuclear signal (Figure 6B).

To determine whether the AtRanBPM protein plays a role in microtubule organization like the truncated version of human RanBPM [37], we studied the colocalization of AtRanBPM with the centrosomal protein, $\gamma$-tubulin. In acentrosomal plant cells, $\gamma$-tubulin is present with dispersed sites of microtubule nucleation in the cytoplasm, with nuclei, and on membranes and microtubules [38]. We found that the Arabidopsis homologue 


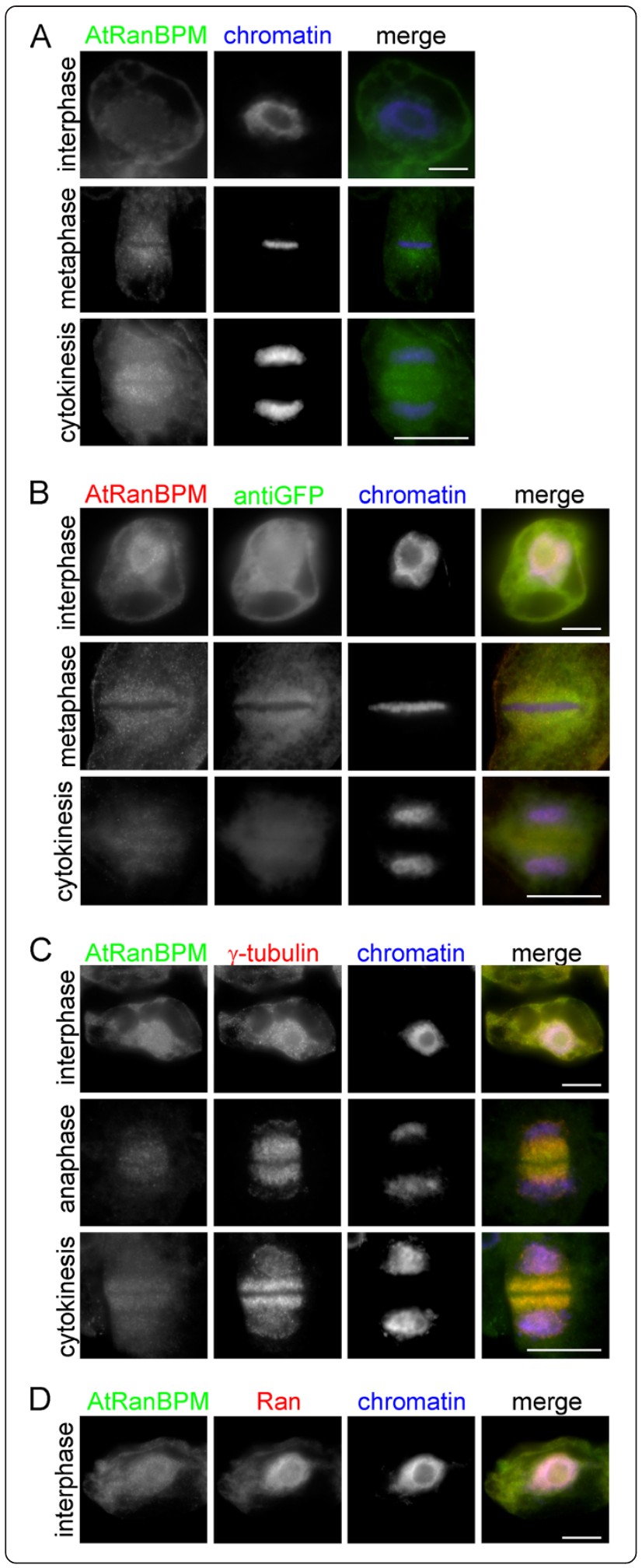

AtRanBPM did not colocalize with $\gamma$-tubulin-positive sites either present with the nuclear envelope or on microtubular arrays (Figure 6C).

As a portion of RanBPM was reported to be associated with the Ran protein in mammalian cells [14] we performed
Figure 6 Immunolocalization of AtRanBPM in Arabidopsis cultured cells. A- Immunolocalization of endogenous AtRanBPM with affinity purified anti-AtRanBPM antibody. Signal for AtRanBPM was cytoplasmic with slight accumulation in the vicinity of nuclei and in dividing cells with slight enrichment in spindle and phragmoplast area. (AtRanBPM - green, chromatin - blue). B- Double immunofluorescence analysis of cells expressing GFP-AtRanBPM confirmed similar localization pattern for expressed GFP protein and for endogenous AtRanBPM protein (Figure 6A) (GFP-AtRanBPM - green, endogenous AtRanBPM - red, chromatin blue). C- Double immunofluorescence analysis of AtRanBPM (green) and $\gamma$-tubulin (red) did not confirm colocalization of both proteins in interphase and in dividing cells. D- Double immunofluorescence of AtRanBPM (green) and Ran (red). Only a small portion of AtRanBPM is localized in nuclei compared to intensive nuclear signal for Ran protein. Bars $=10 \mu \mathrm{m}$.

double immunofluorescence labelling of AtRanBPM and Ran protein. In cultured cells of Arabidopsis, Ran was localized in the cytoplasm and in nuclei but only a small fraction of AtRanBPM colocalized with nuclear Ran.

\section{Discussion}

RanBPM protein was first characterized in human cells as a centrosomal protein involved in microtubule nucleation which colocalized with $\gamma$-tubulin at centrosomes and at ectopic nucleation sites [37]. The data on RanBPM initiated an investigation of the role of the RanGTPase pathway and its role in chromatin-mediated microtubule nucleation and spindle assembly [39]. However, later it was found that antibody against the $55 \mathrm{kDa}$ form of RanBPM that was characterized by Nakamura et al. [37] did not recognize the full length $90 \mathrm{kDa}$ RanBPM protein. The truncated form of RanBPM (55 kDa) was shown to be an incorrectly translated product of the RanBPM gene and moreover, only the truncated version but not the whole RanBPM molecule was active in microtubule nucleation [14]. The complete RanBPM molecule thus does not show the same properties as the truncated version and it was depicted as a scaffold protein that links and modulates interactions between cell surface receptors and their intracellular signalling pathways $[11,40]$. The molecular mass of plant AtRanBPM (52 kDa) corresponds to that of the truncated RanBPM rather than to the whole human RanBPM molecule. However, the reason for the apparent discrepancy in size of the full length human and plant RanBPM molecules is an insertion of 150 amino acids between CTLH and CRA domains in the plant protein and the presence of a long stretch of proline and glutamine residues on the $\mathrm{N}$-terminal part of human RanBPM molecule.

We found that AtRanBPM maintained the properties of the full length RanBPM of mammals: (i) while the truncated version of human RanBPM is present in 
centrosomal and ectopic microtubule nucleation sites [37], AtRanBPM protein in acentrosomal plant cells did not colocalize with microtubule nucleation sites positive for $\gamma$-tubulin; (ii) proteins of the $\gamma$-tubulin nucleating machinery were not identified by MALDI-MS or LC-MALDI-MS/MS among proteins interacting with AtRanBPM, nor were they found in coexpression databases and a biological role of AtRanBPM other than in processes relating to centrosomes and microtubules was thus suggested; (iii) the presence of highly conserved SPRY, LisH, CTLH and CRA domains in AtRanBPM indicated its function in mediating multiple protein interactions that were described for the whole molecule of human RanBPM [12,14,15].

AtRanBPM protein was not specifically enriched with microtubules in dividing cells or localized in putative microtubule nucleation sites. Subcellular localization of AtRanBPM corresponded to published data on the subcellular localization of the whole RanBPM molecule in mammalian cells $[14,41,42]$. Since a weak nuclear signal, observed for human RanBPM was suggested to be a consequence of over-expression of its tagged version [40], we analysed by immunofluorescence the nuclear localization for endogenous AtRanBPM protein. A smaller portion of AtRanBPM was present in nuclei and the possibility that the nuclear signal observed for GFPAtRanBPM or AtRanBPM-GFP resulted from over expression of GFP fusions was thus excluded. We observed only partial colocalization of Ran and AtRanBPM in nuclei. It would be interesting to address the question whether transport of the AtRanBPM complex between nucleus, perinuclear area and cytoplasm might be regulated by a weak interaction between AtRanBPM and Ran as it was suggested for mammalian RanBPM [14]. Heterogeneity in the localization of mammalian RanBPM might depend on interacting proteins [15]. Conserved domains of RanBPM protein are responsible for interactions and complex formations with a physiologically divergent group of proteins. As reviewed by Suresh et al. [11], RanBPM is a modulator/protein stabilizer, a regulator of transcriptional activity, and has cell cycle and neurological functions. RanBPM interacts with a wide range of receptors [43], acts as a scaffolding protein [44,45], and is involved in signal transduction pathways $[44,46]$, and the apoptotic pathways [47]. Plant homologues of RanBPM belong to genes with unknown functions. We found that the product of AtRanBPM is predominantly a cytoplasmic protein that is a part of protein complexes with a molecular mass of approximately $230-500 \mathrm{kDa}$. A large protein complex of RanBPM was described by Nishitani et al. [14] and Ideguchi et al. [13]. Later the complex was designated as a CTLH complex [15]. Five from eleven CTLH domain-containing proteins that were identified in databases were described as being a part of the CTLH complex and an interaction within the complex via LisH-CTLH domains was suggested [15]. In Saccharomyces cerevisiae all four CTLH domain-containing proteins present in the genome are part of the CTLH-like complex Gid. The Gid complex is suggested to be involved in proteasome-dependent glucose-induced degradation of fructose-1,6-bisphosphatase [16,48]. There are twenty one CTLH-domain containing proteins encoded in the Arabidopsis genome and of these, six proteins were identified in our experiments to form CTLH-like complexes with AtRanBPM protein. Though we identified the same spectrum of AtRanBPM interacting proteins in several independent experiments it cannot be ruled out that the members of CTLH complexes exist and remain to be identified. We found that the antibody raised against a peptide from the AtRanBPM sequence did not work in immunopurification experiments. The immunopurifications performed with a panel of antibodies against the AtRanBPM protein might help to disclose the presence of other putative members of plant CTLH complexes. Alternatively, immunopurification of the CTLH complex or pull down experiments via proteins that copurified with AtRanBPM might extend our knowledge of the composition and protein interactions of newly identified CTLH complexes.

All the members of the CTLH complexes that we identified belong to yet uncharacterized plant LisHCTLH domain-containing proteins. Protein At1g61150 is a homologue of the human Twal protein found in yeast by two-hybrid analysis to interact with RanBPM [12]. LisH, CRA, and RING/U-box domain-containing proteins of unknown function (At3g55070, At4g37880), copurified with AtRanBPM, are homologous to human MAEA and RMD5, respectively, that are members of the CTLH complex annotated by Kobayashi et al. [15]. Similarly, LisH and CTLH domain-containing proteins such as RanBP10 or the WD repeat domain 26 (WDR26), were suggested to be putative members of the CTLH complex [15]. We found that uncharacterized Arabidopsis transducin/WD-40 domain-containing proteins At5g08560 and At5g43920 which copurified with AtRanBPM, are homologues of human WDR26 protein and thus belong to components of the plant CTLH complex. Corresponding to our microscopic data on cytoplasmic and nuclear localizations of AtRanBPM, the members of the human CTLH complex, proteins RanBPM, RMD5, WDR26, Twa1, and MAEA, were shown to have predominantly cytoplasmic and a weaker nuclear localization $[12,15,49]$.

A component of the human CTLH complex, the armadillo repeat-containing protein ARMC8, when exogenously expressed, upregulates the proteolytic degradation of ectopically expressed $\alpha$-catenin, and thus was 
suggested to play an important role in the ubiquitinindependent and proteasome-dependent degradation of $\alpha$-catenin [35]. $\alpha$-Catenin is known to function as a link protein between cadherins and actin-containing filaments of the cytoskeleton [50]; thus a complex might regulate molecular adhesion. We identified Armadillorepeat-containing protein (At3g08947) among proteins copurified with AtRanBPM but its homology with armadillo repeat ARMC8, a member of the human CTLH complex, was not proven. However, we found that expression of copurified protein At4g37880, a homologue of RMD5 protein, correlated strongly with expression of the armadillo repeat-containing protein At3g01400, one of the homologues of ARMC8, and with expression of the plant adhesion molecule RabGAP/TBC domaincontaining protein At3g02460 (ATTED-II, [51]). It is tempting to speculate whether similar function in connecting external signals in adhesion with the cell centre analogous to the cadherin and catenin pathway in animals might exist for plant CTLH complexes. However, further experimental data are needed to prove this hypothetical function of the CTLH complexes in plants.

Besides copurification with CTLH complex proteins, we found that plant AtRanBPM interacts with Yippee proteins (At5g53940, At2g40110) and Yippee-like protein (At3g08890). It was shown in human cells that RanBPM binds members of YPEL (Yippee like) family of proteins involved in a cell division-related functions [10]. Our data showed that binding with Yippee proteins might present one of the AtRanBPM multiple protein interactions in plant cells too.

\section{Conclusions}

RanBPM protein was shown to have numerous apparently functionally unrelated protein interactors, including membrane receptors, cytoplasmic and nuclear proteins $[12,15]$. This suggests its role in a variety of cellular processes. Our biochemical and proteomic studies showed that AtRanBPM is a component of plant homologues of CTLH complexes. The fact that CTLH complexes are present in mammals, yeast and in plants suggests their structural conservation in evolution. CTLH complexes thus might represent a module with important but not fully understood biological functions.

\section{Methods}

\section{Plant material}

Arabidopsis thaliana (L.) Heynh. plants, ecotype Columbia were used. Sterilized seeds were sown and stratified at $4{ }^{\circ} \mathrm{C}$ for 2 days, then grown under $8 \mathrm{~h}$ of light, $16 \mathrm{~h}$ of darkness at $20{ }^{\circ} \mathrm{C}$ and after 4 weeks, under $16 \mathrm{~h}$ of light and $8 \mathrm{~h}$ of darkness. Seeds were cultivated on half-strength Murashige and Skoog (MS) medium (Duchefa), supplemented with $0.25 \mathrm{mM}$ MES, 1\% saccharose and 1\% phytoagar. Transformed seedlings were selected as described in Harrison et al. [52]. Arabidopsis thaliana suspension cultures were cultured as described in Drykova et al. [38].

\section{Molecular cloning of AtRanBPM}

AtRanBPM coding sequence [EMBL:AEE31799] was obtained by PCR amplification using Arabidopsis thaliana cDNA as template and Platinum Pfx DNA Polymerase (Invitrogen). The PCR primers with attB sites (underlined) were designed according to the manufacturer's intruction, forward 5' - GGGGACAAGTTTGTACAAAAAAGCAGGCTTCATGAACTCTTCACCACCACCG - 3, reverse $5^{\prime}$ - GGGGACCACTTTGTACAAGAAAGCTG GGTCTTAGTCTCCATTCAGTGACCGCCTTTC - 3' for $\mathrm{N}$-terminal GFP fusion and for $\mathrm{C}$-terminal GFP fusion forward 5' - GGGGACAAGTTTGTACAAAAAAGCAG GCTTCATGAACTCTTCACCACCACCG - 3', reverse $5^{\prime}$ - GGGGACCACTTTGTACAAGAAAGCTGGGTCT TAGTCTCCATTCAGTGACCGCCTTTC - 3'. PCR products were isolated using QIAquick gel extraction kit (Qiagen) and afterwards cloned by Gateway technology (Invitrogen). Gateway binary vectors pK7WGF2,0 for Nterminal GFP fusion, pMDC43 for C-terminal GFP fusion [53] were used.

\section{Stable transformation of cell suspension cultures and plants}

Arabidopsis suspension cultures of Landsberg erecta ecotype stably expressing GFP-AtRanBPM or transiently expressing AtRanBPM-GFP were prepared according to the protocol of Mathur et al. [30]. Arabidopsis plants Landsberg erecta ecotype were transformed by the floral-dip method [31].

\section{Preparation of protein extracts and its fractionation by differential centrifugation}

Cultured suspension cells or seedlings of Arabidopsis thaliana were ground in liquid nitrogen and thawed in extraction buffer in ratio 1:1 weight to volume. Extraction buffer was $50 \mathrm{mM} \mathrm{Na}$-Hepes, pH 7.5, $75 \mathrm{mM} \mathrm{NaCl}$, $1 \mathrm{mM} \mathrm{MgCl}$, 1 mM EGTA, $1 \mathrm{mM}$ DTT supplemented with inhibitors of proteases and phosphatases according to Drykova et al. [38] with increased concentration of 4-(2-aminoethyl)benzenesulfonyl fluoride hydrochloride to $2 \mathrm{mM}$ and $\beta$-glycerophosphate to $60 \mathrm{mM}$. Crude extracts were centrifuged at $10,000 \mathrm{~g}$ for $10 \mathrm{~min}$ at $4{ }^{\circ} \mathrm{C}$; obtained supernatants $\mathrm{S} 10$ were subsequently centrifuged at $20,000 \mathrm{~g}$ for $0.5 \mathrm{~h}$ at $4{ }^{\circ} \mathrm{C}$ to gain supernatants $\mathrm{S} 20$ and pellets P20. Supernatants S20 were then centrifuged at $100,000 \mathrm{~g}$ for $1 \mathrm{~h}$ at $4{ }^{\circ} \mathrm{C}$ when S100 were needed. Supernatants S100 were centrifuged at 200,000 $g$ for $1.5 \mathrm{~h}$ at $4{ }^{\circ} \mathrm{C}$ to gain supernatants S200 and pellets P200 when needed. For differential centrifugation, pellets were 
resuspended in a volume of extraction buffer equal to the volume of the corresponding supernatants; equal sample volumes were loaded on SDS-PAGE gel.

\section{Immunoprecipitation of GFP-AtRanBPM}

Cell extracts S20 $(\sim 4 \mathrm{mg}$ protein $/ \mathrm{mL})$ were used directly or solubilized by $1 \%$ NP-40 (Calbiochem) for $1 \mathrm{~h}$ at $4{ }^{\circ} \mathrm{C}$. Immunoprecipitations of GFP-AtRanBPM protein were then performed using GFP-Trap_A (ChromoTek) according to the manufactures' instructions. GFP immunopurification from extracts S20 of wild type Ler Arabidopsis culture was used as a negative control.

\section{Protein digestion and mass spectrometry}

The analysed protein bands were cut out of the Agstained SDS-PAGE gels, trypsin-digested and the proteins were identified by peptide mass mapping as described elsewhere [54]. Alternatively, eluates from the GFP trap were denatured in $8 \mathrm{M}$ urea and digested overnight using trypsin. After desalting the resulting peptides were separated using the Ultimate HPLC system (LC Packings) on a Magic C18AQ column. The eluent from the column was spotted directly onto PAC-target using a ProteineerFC spotting device (Bruker Daltonics). Automatic MALDI MS/MS analyses were performed on an Ultraflex III TOF/TOF (Bruker Daltonics) and the proteins were identified by searching MS/MS spectra against $A$. thaliana subset of NCBI database using the in-house Mascot program (Matrixscience). The high resolution MALDI spectra were acquired on an APEXQe 9.4 T FTMS instrument (Bruker Daltonics).

\section{Electrophoresis and immunoblotting}

Protein samples after addition of appropriate sample buffer were separated either on 10\% SDS-PAGE gels [55] or on 3-10\% non-denaturing PAGE gels as described previously in Drykova et al. [38]. Proteins were transferred onto $0.45 \mu \mathrm{m}$ polyvinylidene fluoride membranes (Millipore) by wet electroblotting and immunodetected with appropriate antibody. Anti-AtRanBPM antibody was designed against peptide of AtRanBPM amino acid sequence (CSTNPNKKDVQRS), raised in rabbits and purified with immunogenic peptide coupled to protein A sepharose beads (GeneScript) and used in dilution 1:100 for Western blotting from non-denaturating PAGE, 1:300-1:500 for Western blotting from SDS-PAGE. Anti-GFP ab290 (Abcam) was diluted 1:2,000. Secondary anti-Rabbit IgG ECL Antibody, HRP Conjugated (GE Healthcare) was diluted 1:10,000; Super Signal West Pico Chemiluminiscent Substrate (Thermal Scientific) was used according to manufacturer's instructions. Alternatively, SDS-PAGE gels were stained with silver, and the selected protein bands analysed by MALDI-MS.

\section{Immunofluorescence}

Arabidopsis thaliana suspension cultures were fixed for 30 min using 3.7\% paraformaldehyde and processed for immunofluorescence labelling according to Binarová et al. [56]. Primary antibodies used: mouse anti- $\alpha-$ tubulin monoclonal antibody DM1A (Sigma) diluted 1:500, mouse monoclonal anti- $\gamma$-tubulin TU-32 (kindly provided by Pavel Dráber, IMG, Prague, Czech Republic) diluted 1:10, mouse anti-GFP antibody (Abcam) diluted 1:1,000, and mouse antibody against human Ran protein (BD Transduction Laboratories) diluted 1:1,000. AntiAtRanBPM antibody was used in a dilution 1:1,000, antirabbit or anti-mouse secondary antibodies DyLight 488 or DyLight 549 (Jackson Immuno Research Laboratories) were diluted 1:250. DNA was stained with DAPI.

\section{Microscopy}

Microscopical analysis was performed on an IX81 motorized inverted research microscope CellR (Olympus) equipped with DSU (Disk Scanning Unit) and digital monochrome CCD camera CCD-ORCA/ER. To avoid filter crosstalk, fluorescence was detected using HQ 480/40 exciter and HQ 510/560 emitter filter cubes for DyLight 488 and HQ 545/30 exciter and HQ 610/75 emitter filter cubes for DyLight 549 (both AHF AnalysenTechnique). Confocal images were taken on Olympus FluoView FV1000 based on IX81 microscope using PLAPO 100x/ 1.45 and UPLSAPO 60x/1.35 objectives. GFP was excited by $473 \mathrm{~nm}$ solid state laser and its emission was detected from 485 to $545 \mathrm{~nm}$. Images were processed and analysed using CellR and FV10-ASW (Olympus). Adobe Photoshop 7.0 was used for preparation of figures.

\section{Database search}

To identify putative domains, SMART (Simple Modular Architecture Research Tool, [57], http://smart.emblheidelberg.de/) and Pfam ([58], http://pfam.sanger.ac. $\mathrm{uk} /$ ) databases were used. Multiple sequence alignment was performed by ClustalX2 [59] and evaluation of similarity and identity was done by WU-BLAST (Basic Local Alignment Search Tool, [22], http://www.ebi.ac. uk/Tools/sss/wublast/). Schematic drawings of protein structure were prepared in DOG (Domain Graph, [60]) and MyDomains (Prosite, http://prosite.expasy.org/cgi-bin/ prosite/mydomains/). Search for putative interaction sites in AtRanBPM amino acid sequence was done in ELM (The eukaryotic linear motif resource for functional sites in proteins, [28], http://elm.eu.org/) and SumoSP 2.0 (Sumoylation sites prediction [29], http://sumosp.biocuckoo.org/). Phylogenetic relationships were analysed in MEGA 5.02 (Molecular evolutionary genetic analysis, [23]). Genes with similar expression pattern to AtRanBPM were identified in publically available databases ATTED-II ([51], http://atted. jp/) and AraNet ([36], http://www.functionalnet.org/aranet/). 
Putative interactors were analysed by Arabidopsis eFP browser ([32], http://bar.utoronto.ca/efp/cgi-bin/efpWeb.cgi) and Genevestigator ([33], https://www.genevestigator.com/ gv/plant.jsp). The information and phylogenetic relationships of human CTLH complex with similar complexes from other species were found in CORUM ([34], http://mips. helmholtz-muenchen.de/genre/proj/corum/).

\section{Additional files}

\section{Additional file 1: Multiple sequence alignment of AtRanBPM}

plant homologues. Sequence alignment of Arabidopsis RanBPM with Ricinus communis, Vitis vinifera, Populus trichocarpa, Sorghum bicolor, Oryza sativa and Zea mays homologues. Alignment was done using ClustalX2 software [59]. Sequence data of this alignment can be found at accession numbers [Swiss-Prot:F4HYD7] for At1g35470, [Swiss-Prot:B9S762] for R. communis, [Swiss-Prot:F6HWC3] for $V$. vinifera, [Swiss-Prot:B9MWC1] for P. trichocarpa, [Swiss-Prot:C5XUT1] for S. bicolor, [Swiss-Prot:Q6Z183] for O. sativa, [Swiss-Prot:B6UAR9] for Z. mays.

Additional file 2: Identities and similarities of conserved domains between Arabidopsis RanBPM and its human homologues. Sequence alignment was done for conserved domains SPRY (A), LisH (B), CTLH (C) and CRA (D) of full-sized $90 \mathrm{kDa}$ form (RanBPM_90) and 55 kDa form (RanBPM_55) of human RanBPM protein, and RanBP10 with Arabidopsis RanBPM using ClustalX2 software [59]. Sequence data of this alignment can be found at accession numbers [Swiss-Prot:Q6VN20] for RanBP10, [Swiss-Prot: Q96S59] for $90 \mathrm{kDa}$ RanBPM and [EMBL:BAA23216] for $55 \mathrm{kDa}$ RanBPM. The sequence of the SPRY domain was encountered from the 99 amino acids in AtRanBPM sequence. E- Levels of identities and similarities in amino acid composition of conserved domains between AtRanBPM and its human homologues RanBPM and RanBP10.

Additional file 3: Phylogenetic analysis of AtRanBPM and its homologues from other eukaryotic species. The tree was constructed by the neighbor-joining method with the MEGA 5.05 software [23]. Branch numbers represent the percentage of bootstrap values in 1000 sampling replicates. The protein accession numbers are [Swiss-prot:F4HYD7] for AtRanBPM At1g35470, [Swissprot: Q9SMS1] At4g09340 (segmental genome duplication of chromosome 1), [Swiss-Prot:B9S762] for R. communis, [Swiss-Prot: F6HWC3] for $V$. vinifera, [Swiss-Prot:B9MWC1] for $P$. trichocarpa, [Swiss-Prot:C5XUT1] for S. bicolor, [Swiss-Prot:Q6Z183] for O. sativa, [Swiss-Prot:B6UAR9] for Z. mays, [Swiss-prot: Q6VN20] for human RanBP10, [Swiss-prot: A3KMV8] for RanBP10 from Bos taurus, [Swissprot: B5LX41] for RanBP10 from Felis catus, [Swiss-prot: Q6VN19] for RanBP10 from Mus musculus, [Swiss-prot: Q1LUS8] for RanBP10 from Danio rerio, [Swiss-prot: Q9PTY5] for RanBP9 from Xenopus laevis, [Swiss-prot: Q96S59] for human RanBP9, [Swiss-prot: P69566] for RanBP9 from Mus musculus, [Swiss-prot: Q4Z8K6] for RanBP9/10 from Drosophila melanogaster and [Swiss-prot: P53076] for Gid1/ Vid30 homologue from Saccharomyces cerevisiae. Distance bars are given bottom left and bootstrap values are indicated at the nodes.

Additional file 4: Immunopurification of GFP-AtRanBPM protein Immunopurification of GFP-AtRanBPM from extracts of GFP-AtRanBPM expressing cell cultures (IP GFP-RanBPM). GFP immunopurification from extracts of wild type Ler Arabidopsis cells (IP WT) was used as a negative control. A- Proteins were silver stained after separation on SDS-PAGE. Bands corresponding to MW similar of the proteins copurified with GFP-AtRanBPM (IP GFP-RanBPM) were not present in the negative control (IP WT). B- Signal for AtRanBPM was absent in the negative control (IP WT) after detection with anti-AtRanBPM antibody on Western blots. C- Proteins identified by MALDI-MS in negative control (IP WT in A) were background contamination.
Additional file 5: Identities and similarities between proteins copurifying with AtRanBPM and human CTLH complex members. Identities and similarities between Arabidopsis and human proteins were analysed in WU-BLAST.

Additional file 6: Additional proteins copurified with AtRanBPM. The proteins were identified by LC-MALDI-MS/MS and the identity of the matched peptides was confirmed by high-resolution MALDI-FTMS with mass accuracy below $1 \mathrm{ppm}$.

Additional file 7: GFP-AtRanBPM in Arabidopsis root cells. AtRanBPM-GFP signal is dynamic and moving with the cytoplasmic stream.

Additional file 8: Cellular localization of C-terminal GFP and N-terminal GFP AtRanBPM fusion proteins. Cells of Arabidopsis expressing C-terminal GFP AtRanBPM (AtRanBPM-GFP) showed weak cytoplasmic and nuclear signal and accumulation of perinuclear GFP signal similarly as observed for N-terminal GFP AtRanBPM (GFP-AtRanBPM).

\section{Abbreviations}

CRA: CT11-RanBPM; CTLH: C-terminal to LisH; GAP: GTPase activating protein; GEF: Guanine nucleotide exchange factor; GFP: Green fluorescent protein; Gid/Vid proteins: Glucose-induced degradation/vacuole-induced degradation proteins; LC: Liquid chromatography; LisH: Lissencephaly type-1-like homology; RanBP: Ran binding protein; RanBP1: Ran binding protein1; RanBPM: Ran binding protein in microtubule organizing centre; SPRY: SPla and Ryanodine receptor; Scorpin family: SPRY-containing Ran binding protein family.

\section{Competing interests}

The authors declare that they have no competing interests.

\section{Acknowledgements}

We thank Dr. Tomáš Takáč for preliminary confocal analyses. This work was supported by grants 204/07/1169, 204/09/P155, and P501/12/2333 from the Grant Agency of the Czech Republic, grant No. CZ.1.05/2.1.00/01.0007 Centre of the Region Haná for Biotechnological and Agricultural Research for ET and BP, IGA UP PrF/2012/001 for ET, grants LC06034 and LC545 from Ministry of Education, Youth and Sports of Czech Republic, and grant IAA500200719 from Grant Agency of the Czech Academy of the Sciences.

\section{Author details}

${ }^{1}$ Centre of the Region Haná for Biotechnological and Agricultural Research, Institute of Experimental Botany AS CR, v.v.i., Sokolovská 6, Olomouc 772 00, Czech Republic. ${ }^{2}$ Institute of Experimental Botany, AS CR, v.v.i., Sokolovská 6 , 772 00, Olomouc, Czech Republic. ${ }^{3}$ Institute of Microbiology, AS CR, v.v.i., Vídeňská 1083, 142 20, Prague 4, Czech Republic.

\section{Authors' contributions}

ET performed databases search, in vivo microscopical analyses and wrote the manuscript. VC carried out immunolocalization and microscopy, and participated in writing the manuscript. LK performed biochemical analysis, isolation of protein complexes and confocal microscopy, BP performed cloning and together with LV, created stably transformed cell lines and plants of Arabidopsis thaliana, and performed in vivo microscopical analyses. PH performed proteomic analyses. GK undertook immunopurification of protein complexes. PB designed and coordinated experimental plans, and wrote the manuscript. All authors read and approved the final manuscript.

Received: 20 December 2011 Accepted: 7 June 2012

Published: 7 June 2012

\section{References}

1. Vetter $\mathbb{R}$, Wittinghofer $A$ : The guanine nucleotide-binding switch in three dimensions. Science 2001, 294:1299-1304.

2. Weis K: Nucleocytoplasmic transport: cargo trafficking across the border. Curr Opin Cell Biol 2002, 14:328-335.

3. Haizel T, Merkle T, Pay A, Fejes E, Nagy F: Characterization of proteins that interact with the GTP-bound form of the regulatory GTPase Ran in Arabidopsis. Plant J 1997, 11:93-103. 
4. Pay A, Resch K, Frohnmeyer H, Fejes E, Nagy F, Nick P: Plant RanGAPs are localized at the nuclear envelope in interphase and associated with microtubules in mitotic cells. Plant J 2002, 30:699-709.

5. Kim SH, Roux SJ: An Arabidopsis Ran-binding protein, AtRanBP1c, is a co-activator of Ran GTPase-activating protein and requires the C-terminus for its cytoplasmic localization. Planta 2003, 216:1047-1052

6. Baloğlu MC, Zakharov FN, Öktem HA, Yücel AM: Molecular cloning, characterization, and expression analysis of a gene encoding a Ran binding protein (RanBP) in Cucumis melo L. Turk J Biol 2011, 35:387-397.

7. Cho HK, Park JA, Pai HS: Physiological function of NbRanBP1 in Nicotiana benthamiana. Mol Cells 2008, 26:270-277.

8. Wang D, Li Z, Schoen SR, Messing EM, Wu G: A novel MET-interacting protein shares high sequence similarity with RanBPM, but fails to stimulate MET-induced Ras/Erk signaling. Biochem Biophys Res Commun 2004, 313:320-326.

9. Dansereau D, Lasko P: RanBPM regulates cell shape, arrangement, and capacity of the female germline stem cell niche in Drosophila melanogaster. J Cell Biol 2008, 182:963-977.

10. Hosono K, Noda S, Shimizu A, Nakanishi N, Ohtsubo M, Shimizu N Minoshima S: YPEL5 protein of the YPEL gene family is involved in the cell cycle progression by interacting with two distinct proteins RanBPM and RanBP10. Genomics 2010, 96:102-111.

11. Suresh B, Ramakrishna S, Baek KH: Diverse roles of the scaffolding protein RanBPM. Drug Discov Today 2012, 17:379-387.

12. Umeda M, Nishitani H, Nishimoto T: A novel nuclear protein, Twa1, and Muskelin comprise a complex with RanBPM. Gene 2003, 303:47-54.

13. Ideguchi H, Ueda A, Tanaka M, Yang J, Tsuji T, Ohno S, Hagiwara E, Aoki A, Ishigatsubo $Y$ : Structural and functional characterization of the USP11 deubiquitinating enzyme, which interacts with the RanGTP-associated protein RanBPM. J Biochem 2002, 367:87-95.

14. Nishitani H, Hirose E, Uchimura Y, Nakamura M, Umeda M, Nishii K, Mori N, Nishimoto T: Full-sized RanBPM CDNA encodes a protein possessing a long stretch of proline and glutamine within the N-terminal region, comprising a large protein complex. Gene 2001, 272:25-33.

15. Kobayashi N, Yang J, Ueda A, Suzuki T, Tomaru K, Takeno M, Okuda K, Ishigatsubo Y: RanBPM, Muskelin, p48EMLP, p44CTLH, and the armadillorepeat proteins $A R M C 8 a$ and $A R M C 8 \beta$ are components of the CTLH complex. Gene 2007, 396:236-247.

16. Regelmann J, Schutz T, Josupeit FS, Horak J, Rose M, Entian K, Thumm M, Wolf DH: Catabolite Degradation of Fructose-1, 6-bisphosphatase in the Yeast Saccharomyces cerevisiae: A Genome-wide Screen Identifies Eight Novel GID Genes and Indicates the Existence of Two Degradation Pathways. Mol Biol Cell 2003, 14:1652-1663.

17. Tomaru K, Ueda A, Suzuki T, Kobayashi N, Yang J, Yamamoto M, Takeno M, Kaneko T, Ishigatsubo Y: Armadillo Repeat Containing 8alpha Binds to HRS and Promotes HRS Interaction with Ubiquitinated Proteins. Open Biochem J 2010, 4:1-8.

18. Aquea F, Matte JP, Gutiérrez F, Rico S, Lamprecht M, Sánchez C, ArceJohnson P: Molecular characterization of a Trithorax-group homologue gene from Pinus radiata. Plant Cell Rep 2009, 28:1531-1538.

19. Gao X, Chen Z, Zhang J, Li X, Chen G, Li X, Wu C: OsLIS-L1 encoding a lissencephaly type-1-like protein with WD40 repeats is required for plant height and male gametophyte formation in rice. Planta 2012, 354:713-727.

20. Proost $S$, Van Bel M, Sterck L, Billiau K, Van Parys T, Van de Peer $Y$, Vandepoele K: PLAZA: a comparative genomics resource to study gene and genome evolution in plants. Plant Cell 2009, 21:3718-3731.

21. Swarbreck D, Wilks C, Lamesch P, Berardini TZ, Garcia-Hernandez M, Foerster H, Li D, Meyer T, Muller R, Ploetz L, Radenbaugh A, Singh S, Swing V, Tissier C, Zhang P, Huala E: The Arabidopsis Information Resource (TAIR): gene structure and function annotation. Nucleic Acids Res 2008, 36:D1009-14.

22. Lopez R: WU-Blast2 server at the European Bioinformatics Institute. Nucleic Acids Res 2003, 31:3795-3798.

23. Tamura K, Peterson D, Peterson N, Stecher G, Nei M, Kumar S: MEGA5: Molecular Evolutionary Genetics Analysis using Maximum Likelihood, Evolutionary Distance, and Maximum Parsimony Methods. Mol Biol Evol 2011, 28:2731-2739.

24. Ponting C, Schultz J, Bork P: SPRY domains in ryanodine receptors (Ca2 +-release channels). Trends Biochem Sci 1997, 5:2-3.
25. Rhodes D, de Bono B, Trowsdale J: Relationship between SPRY and B30.2 protein domains. Evolution of a component of immune defence? Immunology 2005, 116:411-417.

26. Emes RD, Ponting CP: A new sequence motif linking lissencephaly, Treacher Collins and oral-facial-digital type 1 syndromes, microtubule dynamics and cell migration. Hum Mol Genet 2001, 10:2813-2820.

27. Menon RP, Gibson TJ, Pastore A: The C Terminus of Fragile X Mental Retardation Protein Interacts with the Multi-domain Ran-binding Protein in the Microtubule-organising Centre. J Mol Biol 2004, 43-53.

28. Puntervoll P: ELM server: a new resource for investigating short functional sites in modular eukaryotic proteins. Nucleic Acids Res 2003, 31:3625-3630

29. Ren J, Gao X, Jin C, Zhu M, Wang X, Shaw A, Wen L, Yao X, Xue Y: Systematic study of protein sumoylation: Development of a site-specific predictor of SUMOsp 2.0. Proteomics 2009, 9:3409-3412.

30. Mathur J, Csaba K: Establishment and Maintenance of Cell Suspension Cultures. In Methods in Molecular Biology. Volume 82. Arabidopsis protocols. Edited by Martinéz-Zapater J, Salinas J. Totowa: Humana Press; 1997:27-30

31. Clough SJ, Bent F: Floral dip: a simplified method for Agrobacteriummediated transformation of Arabidopsis thaliana. Plant J 1998, 16:735-743

32. Winter D, Vinegar B, Nahal H, Ammar R, Wilson GV, Provart NJ: An "Electronic Fluorescent Pictograph" browser for exploring and analyzing large-scale biological data sets. PLoS One 2007, 2:e718.

33. Hruz T, Laule O, Szabo G, Wessendorp F, Bleuler S, Oertle L, Widmayer P, Gruissem W, Zimmermann P: Genevestigator v3: a reference expression database for the meta-analysis of transcriptomes. Advances in bioinformatics 2008, 2008:420747.

34. Ruepp A, Waegele B, Lechner M, Brauner B, Dunger-Kaltenbach I, Fobo G, Frishman G, Montrone C, Mewes HW: CORUM: the comprehensive resource of mammalian protein complexes-2009. Nucleic Acids Res 2010, 38:D497-501.

35. Suzuki T, Ueda A, Kobayashi N, Yang J, Tomaru K, Yamamoto M, Takeno M, Ishigatsubo Y: Proteasome-dependent degradation of alpha-catenin is regulated by interaction with ARMc8alpha. J Biochem 2008, 411:581-591.

36. Lee I: Ambaru Bi, Thakkar P, Marcotte EM, Rhee SY: Rational association of genes with traits using a genome-scale gene network for Arabidopsis thaliana. Nature Biotechnol 2010, 28:149-156.

37. Nakamura M, Masuda H, Horii J, Kuma Kl, Yokoyama N, Ohba T, Nishitani H, Miyata T, Tanaka M, Nishimoto T: When overexpressed, a novel centrosomal protein, RanBPM, causes ectopic microtubule nucleation similar to gamma-tubulin. J Cell Biol 1998, 143:1041-1052.

38. Dryková D, Cenklová V, Sulimenko V, Volc J, Dráber P, Binarová P: Plant gamma-Tubulin Interacts with alpha-Tubulin Dimers and Forms Membrane-Associated Complexes. Plant Cell 2003, 15:465-480.

39. Heald R, Weis K: Spindles get the Ran around. Cell 2000, 8924:1-4.

40. Murrin LC, Talbot JN: RanBPM, a scaffolding protein in the immune and nervous systems. J Neuroimmune Pharm 2007, 2:290-295.

41. Zou Y, Lim S, Lee K, Deng X, Friedman E, Chem JB: Serine/Threonine Kinase Mirk/Dyrk1B Is an Inhibitor of Epithelial Cell Migration and Is Negatively Regulated by the Met Adaptor Ran-binding Protein M. J Biol Chem 2003, 278:49573-49581.

42. Denti S, Sirri A, Cheli A, Rogge L, Innamorati G, Putignano S, Fabbri M, Pardi R, Bianchi E: RanBPM Is a Phosphoprotein That Associates with the Plasma Membrane and Interacts with the Integrin LFA-1.J Biol Chem 2004, 279:13027-13034.

43. Poirier MB, Laflamme L, Langlois MF: Identification and characterization of RanBPM, a novel coactivator of thyroid hormone receptors. J Mol Endocrinol 2006, 36:313-325.

44. Talbot JN, Skifter DA, Bianchi E, Monaghan DT, Toews ML, Murrin LC, Myron LT: Neuroscience Letters Regulation of mu opioid receptor internalization by the scaffold protein RanBPM. Neurosci Lett 2009, 466:154-158.

45. Puverel S, Barrick C, Dolci S, Coppola V, Tessarollo L: RanBPM is essential for mouse spermatogenesis and oogenesis. Development 2011, 138:2511-2521.

46. Cheng L, Lemmon S, Lemmon V: RanBPM is an L1-interacting protein that regulates $\mathrm{L} 1$-mediated mitogen-activated protein kinase activation. J Neurochem 2005, 94:1102-1110. 
47. Atabakhsh E, Bryce DM, Lefebvre KJ, Schild-Poulter C: RanBPM has proapoptotic activities that regulate cell death pathways in response to DNA damage. Mol Cancer Res 2009, 7:1962-1972.

48. Santt O, Pfirrmann T, Braun B, Juretschke J, Kimmig P, Scheel H, Hofmann K, Thumm M, Wolf DH: The Yeast GID Complex, a Novel Ubiquitin Ligase (E3) Involved in the Regulation of Carbohydrate Metabolism. Mol Biol Cell 2008, 19:3323-3333.

49. Zhu Y, Wang Y, Xia C, Li D, Li Y, Zeng W, Yuan W, Liu H, Zhu C, Wu X, Liu M: WDR26: a novel Gbeta-like protein, suppresses MAPK signaling pathway. J Cell Biochem 2004, 93:579-87.

50. Kobielak A, Pasolli HA, Fuchs E: Mammalian formin-1 participates in adherens junctions and polymerization of linear actin cables. Nature Cell Biol 2004, 6:21-30.

51. Obayashi T, Nishida K, Kasahara K, Kinoshita K: ATTED-II updates: conditionspecific gene coexpression to extend coexpression analyses and applications to a broad range of flowering plants. Plant Cell Physiol 2011 , 52:213-219.

52. Harrison SJ, Mott EK, Parsley K, Aspinall S, Gray JC, Cottage A: A rapid and robust method of identifying transformed Arabidopsis thaliana seedlings following floral dip transformation. Plant Methods 2006, 2:19-25.

53. Karimi M, Inzé D, Depicker A: GATEWAY vectors for Agrobacteriummediated plant transformation. Trends Plant Sci 2002, 7:193-195.

54. Halada P, Man P, Grebenova D, Hrkal Z, Havlicek V: Identification of HL60 proteins affected by 5 -aminolevulinic acid-based photodynamic therapy using mass spectrometric approach. Collect Czech Chem Commun 2001, 66:1720-1728

55. Laemmli UK: Cleavage of structural proteins during the assembly of the head of bacteriophage T4. Nature 1970, 227:680-685.

56. Binarová P, Cenklová V, Procházková J, Doskočilová A, Volc J, Vrlík M, Bogre L: gamma-Tubulin Is Essential for Acentrosomal Microtubule Nucleation and Coordination of Late Mitotic Events in Arabidopsis. Plant Cell 2006, 18:1199-1212.

57. Letunic I, Doerks T, Bork P: SMART 7: recent updates to the protein domain annotation resource. Nucleic Acids Res 2011, :1-4.

58. Finn RD, Tate J, Mistry J, Coggill PC, Sammut SJ, Hotz HR, Ceric G, Forslund K, Eddy SR, Sonnhammer EL, Bateman A: The Pfam protein families database. Nucleic Acids Res 2008, 36:D281-8.

59. Larkin M, Blackshields G, Brown NP, Chenna R, McGettigan P, McWilliam H, Valentin F, Wallace IM, Wilm A, Lopez R, Thompson JD, Gibson TJ, Higgins DG: Clustal W and Clustal X version 2.0. Bioinformatics 2007, 23:2947-2958.

60. Xao X, Xue Y: DOG 1.0: illustrator of protein domain structures. Cell Res 2009, 19:271-273.

doi:10.1186/1471-2229-12-83

Cite this article as: Tomaštíková et al: Interactions of an Arabidopsis RanBPM homologue with LisH-CTLH domain proteins revealed high conservation of CTLH complexes in eukaryotes. BMC Plant Biology 2012 $12: 83$.

\section{Submit your next manuscript to BioMed Central and take full advantage of:}

- Convenient online submission

- Thorough peer review

- No space constraints or color figure charges

- Immediate publication on acceptance

- Inclusion in PubMed, CAS, Scopus and Google Scholar

- Research which is freely available for redistribution 